\title{
Analysis of Spinopelvic Parameters with L5 as the New Sacrum after Fusion in High-Grade Spondylolisthesis: A Possible Explanation for Satisfactory Results with In-Situ Fusion
}

\author{
Shanmuganathan Rajasekaran, Gurudip Das, Siddharth Narasimhan Aiyer, \\ Rishi Mugesh Kanna, Ajoy Prasad Shetty \\ Department of Orthopaedics and Spine Surgery, Ganga Hospital, Coimbatore, India
}

\begin{abstract}
Study Design: Retrospective case series.
Purpose: To correlate functional outcomes with spinopelvic parameters in patients with high-grade spondylolisthesis (HGS) treated with instrumented in-situ surgery or reduction and fusion

Overview of Literature: Satisfactory functional outcomes are reported with reduction and in-situ fusion strategies in HGS. However, reasons for this are unclear. We hypothesize that following lumbosacral fusion, the L 5 becomes part of the sacrum, which improves spinopelvic parameters, resulting in equivalent functional outcomes in both surgical methods.

Methods: Twenty-six patients undergoing HGS (reduction group A, 13; in-situ group B, 13) were clinically evaluated using the 0swestry Disability Index (ODI), short form-12 (SF-12), and Visual Analogue Scale (VAS) scores. Spinopelvic parameters, including pelvic incidence, pelvic tilt (PT), sacral slope (SS), lumbar lordosis (LL), lumbosacral kyphosis (LSK) angle, and sacrofemoral distance (SFD) were measured preoperatively from S1 and postoperatively from $\mathrm{L} 5$ as the new sacrum at 1 year follow-up. Sagittal alignment was assessed using the sagittal vertical axis.

Results: Both groups were comparable in terms of age, sex, severity of slip, and preoperative spinopelvic parameters $(p>0.05)$. Postoperative VAS, SF-12, and ODI scores significantly improved in both groups $(p<0.05)$. Compared with preoperative values, the mean postoperative PT, SFD, and LSK significantly changed in both groups. In reduction group, PT changed from $26.98^{\circ}$ to $10.78^{\circ}$, SFD from 61.24 to $33.56 \mathrm{~mm}$, and LSK from $74.76^{\circ}$ to $109.61^{\circ}(p<0.05)$. In in-situ fusion group PT changed from $26.78^{\circ}$ to $11.08^{\circ}$, SFD from 62.9 to $36.99 \mathrm{~mm}$, and LSK from $67.23^{\circ}$ to $113.38^{\circ}$ ( $p<0.05$ for all). In both groups, SS and LL did not change significantly $(p>0.05)$. Conclusions: After fusion, the $L 5$ becomes the new sacrum and influences spinopelvic parameters to change favorably. This possibly explains why reduction and in-situ fusion achieve equivalent functional outcomes in HGS.
\end{abstract}

Keywords: Lumbar spine; Spondylolisthesis; Reduction; Spinal fusion

Received Mar 6, 2017; Revised May 11, 2017; Accepted May 26, 2017

Corresponding author: Shanmuganathan Rajasekaran

Department of Orthopaedics, Traumatology and Spine Surgery, Ganga Hospital, 313, Mettupalayam road, Coimbatore 641043, India Tel: +91-422-2485000, Fax: +91-422-2451444, E-mail: rajasekaran.orth@gmail.com 


\section{Introduction}

Two schools of thought pertaining to the treatment strategies for high-grade spondylolisthesis (HGS) are in-situ fusion and reduction of slip and fusion [1-9]. Advocates of reduction with fusion cite several advantages to this technique, including placement of the fusion mass at a biomechanical advantage, improved cosmesis, improvement in gait biologics, restoration of spinal canal dimensions, and spinal sagittal alignment [2-5]. The Spinal Deformity Study Group recently suggested that defining global sagittal plane balance is a key factor in developing treatment algorithms for patients with HGS [10-12]. In patients with HGS, this has provided a compelling rationale to reduce and realign the deformity to restore global spinopelvic balance, especially in those with an unbalanced spine. Although reduction and fusion reestablishes the spatial orientation of the L5 with respect to the S1, this technique has been associated with a significant rate of neurological complications [13-15] with a risk of neurological injury approaching $75 \%$ in one series [5].

In-situ fusion has been advocated in several studies reporting good functional outcomes [6-9]. Despite equivalent clinical outcomes with reduction and fusion, a major concern with in-situ fusion is the inability to restore pelvic parameters to normal. If in-situ fusion can achieve equally
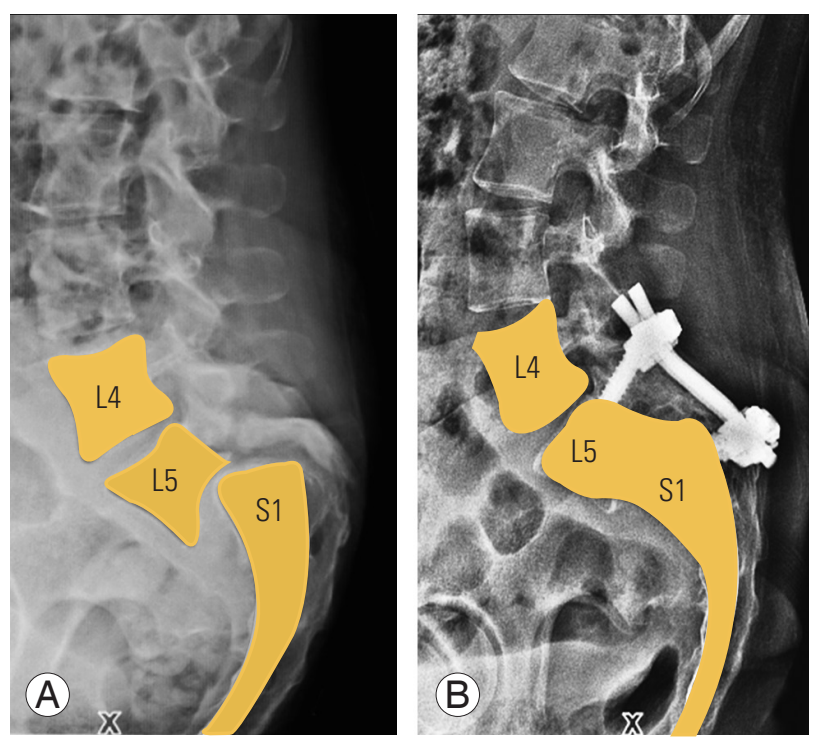

Fig. 1. (A) Preoperative plain radiograph showing the high-grade slip. (B) Postoperative plain radiograph with fusion. The $L 5$ is a part of the pelvis and the top of $L 5$ acts as the new sacrum. This hypothesis is used to calculate all pelvic parameters. good results short-term and long-term, the question arises whether addressing the instability is the key determinant of the outcomes. Achieving good clinical results without addressing restoration of sagittal parameters has not been explained clearly in the literature. When a stable fusion is achieved between the L5 and sacrum, the spinopelvic morphology changes. Hence, postoperative spinopelvic parameters may be measured from the top of the L5 (Fig. 1). Clinical and functional outcomes of patients with HGS treated via in-situ fusion or reduction and fusion were analyzed. Spinopelvic parameters were compared between patients undergoing in-situ fusion and reduction and fusion from the top of the L5 in both groups postoperatively.

\section{Materials and Methods}

Twenty-six consecutive patients who underwent surgery for HGS between August 2009 and August 2013 were retrospectively reviewed. The study was approved by the institute review board at Ganga Hospital, Coimbatore, India (approval no., 20130302) and was conducted in accordance with the ethical standards outlined in the Declaration of Helsinki. Inclusion criteria were patients with grades 3 and 4 spondylolisthesis on preoperative standing whole spine lateral radiographs. Surgery was performed by two operating spine units: one unit performed in-situ
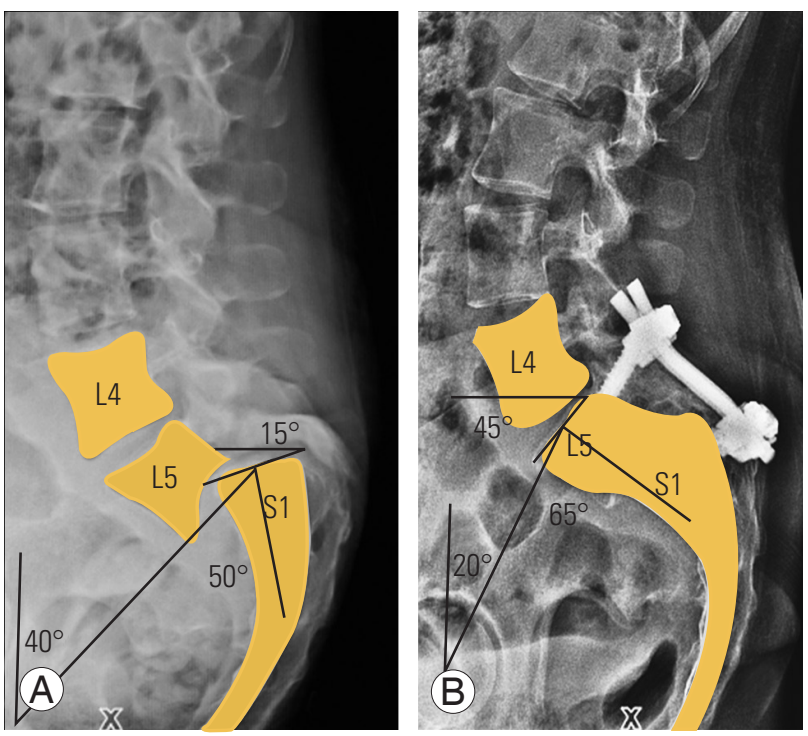

Fig. 2. (A) Measurement of preoperative PI, PT, and SS with S1 as the top of sacrum. (B) Measurement of postoperative PI, PT, and SS with the L5 as the top of new sacrum. Pl, pelvic incidence; PT, pelvic tilt; SS, sacral slope. 
fusion and the other reduction and fusion. The patients studied were divided into two groups depending on the procedure performed: reduction and fusion (group A, $\mathrm{n}=13$ patients) and in-situ fusion (group $\mathrm{B}, \mathrm{n}=13$ patients).

All patients were assessed using standard standing whole spine radiographs. After digitalization of the film and transfer to the picture archiving and communications system server, radiographic measurements were performed using VEPRO software (ver. 7.2; VEPRO GmbH, Pfungstadt, Germany). The following radiographic parameters were studied: pelvic incidence (PI), pelvic tilt (PT), sacral slope (SS), lumbar lordosis (LL), sacrofemoral distance (SFD), and lumbosacral kyphosis (LSK). Standard definition for each pelvic parameter was used for calculation $[12,16]$ (Fig. 2). Dubousset's lumbosacral angle (LSA) was used to measure LSK. Sagittal alignment was assessed using the sagittal vertical axis (SVA). SVA was drawn vertically downward from the $\mathrm{C} 7$. The line passing anterior to the posterior superior corner of the S1 was given a positive value and that passing posterior to the posterior superior corner of the $\mathrm{S} 1$ was given a negative value.

\section{Preoperative pelvic parameter assessment}

Parameters were calculated from the S1 as the top of the sacrum preoperatively in both groups. Preoperative PI, PT, SS, LL, LSK, and SFD for both groups were analyzed

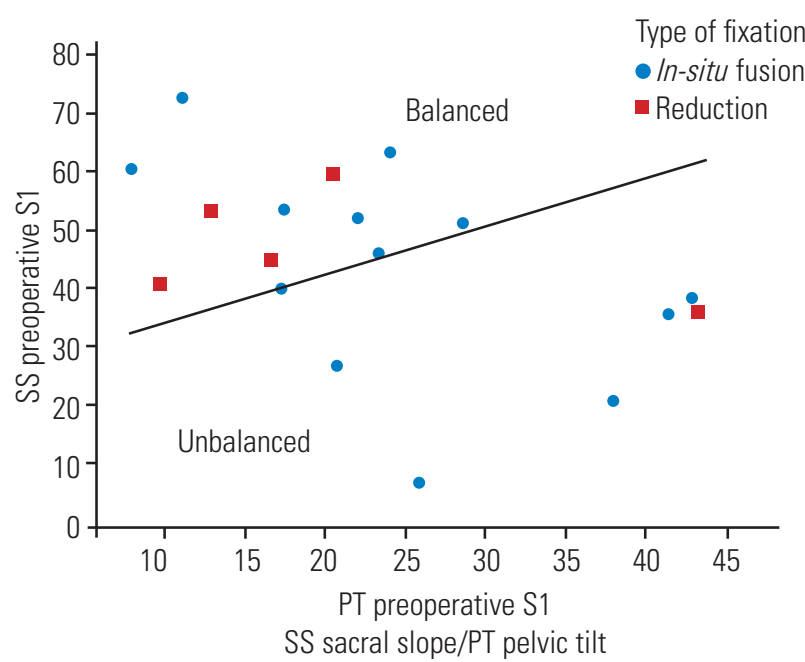

Fig. 3. Graph showing the plot of the preoperative PT on the x-axis and preoperative SS on the $y$-axis with the $S 1$ as the base of the pelvis. Higher PT and lower SS are considered to form the unbalanced pelvis group and lower PT with higher SS form the balanced pelvis group. Square plots indicate patients in group $A$ and dots indicate those in group B. PT, pelvic tilt; SS, sacral slope. to assess if the groups were comparable (Fig. 2). Patients were also assessed using the Spine Deformity Study Group criteria for balanced and unbalanced pelvis [17]. Patients with high PT and low SS were considered to have unbalanced pelvis and those with low PT and high SS were considered to have balanced pelvis (Fig. 3) with both groups having a comparable distribution of balanced and unbalanced pelvis cases.

\section{Postoperative pelvic parameter assessment}

After lumbosacral fusion, the L5 becomes a part of the sacrum. Hence, for postoperative radiological assessment, we calculated pelvic parameters using the L5 superior endplate as the new sacrum and a comparative analysis was performed for both groups (Fig. 2). LL was calculated from the superior endplate of the L1 to the inferior endplate of the L4 postoperatively, considering that the L5 was a part of the pelvis. LSK was preoperatively calculated with lines drawn along the L5 superior endplate and posterior sacral margin. Postoperatively, it was measured with lines drawn along the L4 superior endplate and posterior sacral margin.

\section{Clinical evaluation}

Clinical data evaluation included assessment of Oswestry Disability Index (ODI), Visual Analogue Scale (VAS) score, and short form-12 (SF-12) score preoperatively and at 6 weeks, 12 months, and 24 months. All complications in both groups were noted.

\section{Statistical analysis}

Statistical analysis was conducted with SPSS ver. 17.0 (SPSS Inc., Chicago, IL, USA). Median, range, and nonparametric tests (Wilcoxon signed-rank test) were used for analysis of nonnormally distributed data. Statistical significance was defined as $p<0.05$.

\section{Surgical technique}

1) In-situ fixation

A posterior midline approach was used. Pedicle screws were inserted into the L5 pedicle; transvertebral pedicle screws were passed into the L5 body through the S1 pedicle (Fig. 4). Connecting rods were placed in a delta configuration. No 
reduction of slip was performed. Midline decompression was performed via laminectomy and posterolateral fusion was performed with autologous bone graft.

2) Reduction and fusion

A posterior midline approach was used. The deformity was reduced by preoperative positioning and intraoperative reduction techniques. Temporary pedicle screws were inserted into the cephalic level unilaterally to allow for distraction and reduction of slip. Reduction screws were inserted into the L5 to allow for further reduction of deformity. Decompression was performed using laminectomy and interbody fusion was performed using titanium box cages (Fig. 4).

\section{Results}

We analyzed 26 patients (mean follow-up, 24.5 months [range, 24-36 months]; mean age, 34.1 years [range, 11-52 years]; 2 males and 24 females). All 26 patients presented with low back pain, with additional radiculopathy in 19. No patient had neurologic deficits at presentation. All patients in both groups were comparable in terms of age, sex, severity of slip, preoperative VAS score, ODI score, SF-12 scores, and preoperative spinopelvic parameters $(p>0.05)$.

Mean surgery duration was 185 minutes (range, 145-240 minutes) in group A and 162 minutes (range, 130-195 minutes) in group B. Average blood loss was 564 $\mathrm{mL}$ (range, $450-800 \mathrm{~mL}$ ) and $478 \mathrm{~mL}$ (range, 300-650 $\mathrm{mL}$ ), respectively. Of 13 patients with transient postoperative paresthesias, 8 were in group A and 5 in group B. One patient in group A had transient extensor hallucis longus weakness. None of the patients had any instrumentation failures at the final follow-up.

The mean ODI, SF-12, and VAS scores for groups A
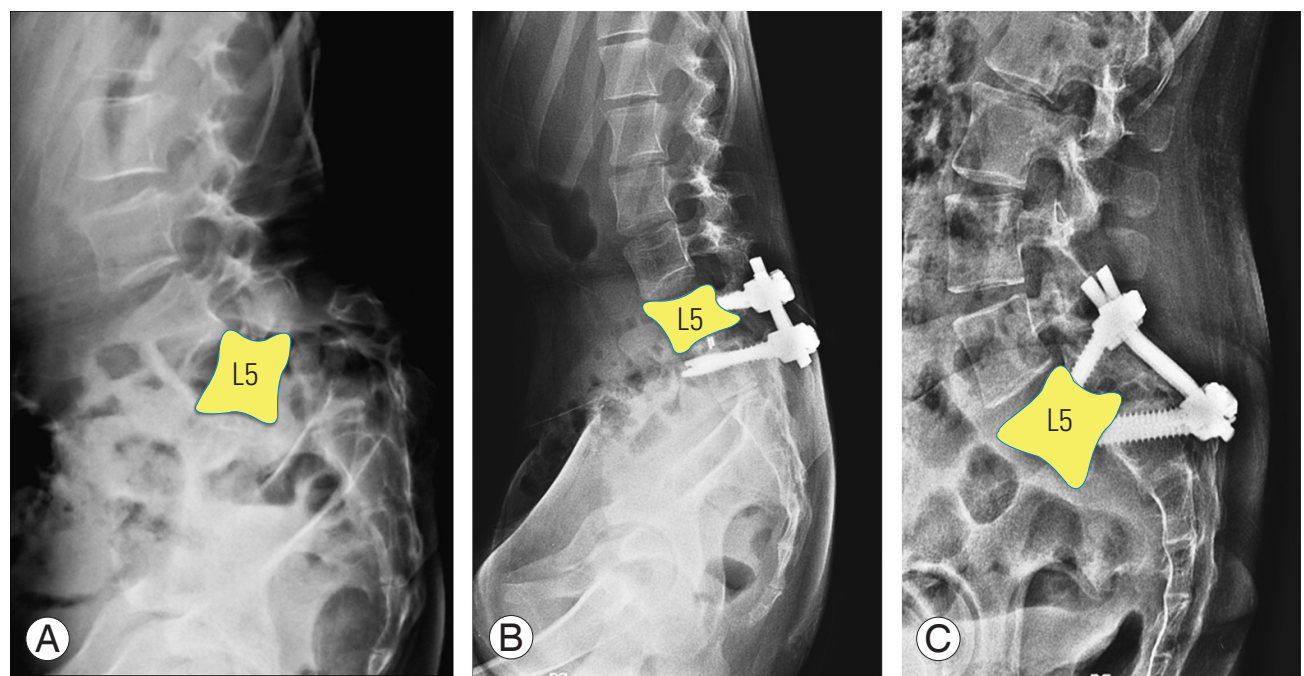

Fig. 4. (A) Preoperative plain radiograph showing a high-grade L5-S1 spondylolisthesis. (B) Postoperative plain radiograph showing reduction of the slip with pedicle screws and interbody fusion using cage. (C) Postoperative plain radiograph showing in-situ fusion using a transpedicular fixation into the L5 in the form of a delta fixation.

Table 1. The functional outcomes for SF-12 scores and ODI scores preoperatively and at 24-month follow-up for the in-situ and reduction group

\begin{tabular}{|c|c|c|c|c|}
\hline \multirow{2}{*}{ Group } & \multicolumn{2}{|c|}{ SF-12 (PCS and MCS) } & \multicolumn{2}{|r|}{ ODI } \\
\hline & Preoperative & Postoperative 24 months & Preoperative & Postoperative 24 months \\
\hline In-situ & $32.02 / 52.06$ & $18.05 / 30.26^{*}$ & 42.76 & $15.4^{*}$ \\
\hline Reduction & $39.39 / 55.66$ & $21.34 / 31.67^{*}$ & 48.52 & $16^{*}$ \\
\hline
\end{tabular}

SF-12, short form-12; ODI, Oswestry Disability Index; PCS, Physical Health Composite Score; MCS, Mental Health Composite Score. " $p<0.05$; indicates significant improvement in the SF-12 and ODI values postoperatively in both groups. However there was no difference between in-situ and reduction groups on comparing the functional outcomes. 
and B were comparable preoperatively $(p>0.05)$ (Tables $1,2)$ and showed significant improvement $(p<0.05)$ at the final follow-up of 24 months with no statistical difference noted between groups. There was no difference in clinical outcome measured with VAS, SF-12, and ODI scores between the groups at any time during follow-up.

\section{Radiological results}

For both groups, mean preoperative values for PI, PT, SS, LL, LSK, and SFD were comparable ( $p>0.05)$, there were significant changes in PT, SFD, and LSK postoperatively in both groups. There were changes noted in SS and LL in both groups, but were insignificant. The radiological pelvic parameter changes for both groups are illustrated in Table 3.

\section{1) Lumbosacral kyphosis}

Mean preoperative LSK was $71^{\circ}$ (range, $35.1^{\circ}-154.7^{\circ}$ ) overall with mean of the in-situ group being $67.23^{\circ}$ and mean of reduction group being $74.6^{\circ}$. The mean in each group was comparable $(p>0.05)$. Postoperatively, when
LSK was measured with the L5 as the top of the sacrum, LSK significantly improved in both groups. The mean LSK changed from $67.23^{\circ}$ to $113.38^{\circ}$ ( $p=0.0002$ ) in-situ group and from $74.6^{\circ}$ to $109.61^{\circ}(p=0.01)$ for reduction group.

\section{2) Sacrofemoral distance}

Mean preoperative SFD was $62.15 \mathrm{~mm}$ (range, 24.9-105.7 $\mathrm{mm}$ ) overall, and 61.24 and $62.9 \mathrm{~mm}$ in groups A and B, respectively $(p>0.05)$. Significant reduction in mean SFD was noted postoperatively in both groups. Mean SFD changed from 61.24 to $33.56 \mathrm{~mm}(p=0.0007)$ and from 62.9 to $36.99 \mathrm{~mm}(p=0.001)$ in groups A and $\mathrm{B}$, respectively.

\section{3) Pelvic tilt}

Mean preoperative PT was $26.88^{\circ}$ (range, $10.2^{\circ}-45.2^{\circ}$ ) with a mean of in-situ group being $26.78^{\circ}$ and mean of the reduction group being $26.98^{\circ}(p>0.05)$. Postoperative changes in PT were significant for both groups. Mean PT for the in-situ group changed from $26.78^{\circ}$ to $11.08^{\circ}$ $(p=0.0005)$ and for the reduction group from $26.98^{\circ}$ to $10.78^{\circ}(p=0.0005)$.

Table 2. The VAS scores for in-situ and reduction groups preoperatively, 6 weeks, 6 months, and at final follow-up

\begin{tabular}{lcccc} 
& \multicolumn{3}{c}{ VAS score } \\
\cline { 2 - 5 } & Preoperative & 6 Weeks & 6 Months & 24 Months \\
In-situ & 7.64 & 4.5 & 3.9 & $3.6^{*}$ \\
Reduction & 7.96 & 5.5 & 4.0 & $3.56^{*}$ \\
\hline
\end{tabular}

VAS, Visual Analogue Scale.

${ }^{*} \mathrm{p}<0.05$; indicates significant improvement in the VAS scores postoperatively in both groups. However there was no difference between in-situ and reduction groups.

Table 3. Showing the pelvic parameters for the reduction and in-situ fusion groups when assessed with S1 as the top of sacrum preoperatively and when measured with L5 as the top of new sacrum post fusion

\begin{tabular}{|c|c|c|c|c|c|c|c|}
\hline \multirow{2}{*}{ Variable } & \multicolumn{3}{|c|}{ Reduction } & \multicolumn{3}{|c|}{ In-situ } & \multirow{2}{*}{$\begin{array}{c}\text { Comparison of } \\
\text { postoperative } L 5^{a}\end{array}$} \\
\hline & Preoperative & Postoperative & $p$-value & Preoperative & Postoperative & $p$-value & \\
\hline Pelvic incidence $\left({ }^{\circ}\right)$ & 69.78 & 41.33 & $0.0002^{*}$ & 68.56 & 61.81 & 0.24 & $0.008^{*}$ \\
\hline Pelvic tilt $\left({ }^{\circ}\right)$ & 26.98 & 10.78 & $0.0005^{*}$ & 26.78 & 11.08 & $0.0005^{*}$ & 0.9 \\
\hline Sacral slope $\left({ }^{\circ}\right)$ & 43.08 & 30.51 & 0.096 & 41.72 & 50.77 & 0.16 & $0.0009^{*}$ \\
\hline Lumbar lordosis $\left({ }^{\circ}\right)$ & 47.92 & 45.88 & 1.00 & 51.46 & 62.65 & 0.08 & $0.0014^{*}$ \\
\hline Sacrofemoral distance (mm) & 61.24 & 33.56 & $0.0007^{*}$ & 62.9 & 36.99 & $0.001^{*}$ & 0.27 \\
\hline Lumbosacral kyphosis $\left({ }^{\circ}\right)$ & 74.76 & 109.61 & $0.01^{*}$ & 67.23 & 113.38 & $0.0002^{*}$ & 0.65 \\
\hline
\end{tabular}

All the angles are measured in degrees and sacrofemoral distance measured in millimetres.

${ }^{*} p<0.05$; indicates statistical significant difference. ${ }^{a}$ Measured spino pelvic parameters between groups. 
4) Pelvic incidence

Mean preoperative PI was $69.17^{\circ}$ (range, $46.10^{\circ}-90.80^{\circ}$; standard deviation $=13.13^{\circ}$ ). The mean PI of in-situ group was $68.56^{\circ}$ and mean of the reduction group was $69.78^{\circ}$ $(p>0.05)$. For the in-situ group comparing PI with L5 as the top of the pelvis after fusion, the mean changed from $68.56^{\circ}$ to $61.81^{\circ}$, which was not significant $(p=0.24)$. For the reduction group, PI changed from $69.78^{\circ}$ to $41.33^{\circ}$ $(p=0.0002)$.

5) Lumbar lordosis and sacral slope

Mean preoperative SS was $42.40^{\circ}$ (range, $15.20^{\circ}-59.60^{\circ}$ ) with a mean of in-situ group being $41.72^{\circ}$ and mean of the reduction group being $43.08^{\circ}(p>0.05)$. For in-situ group, the mean changed from $41.72^{\circ}$ to $50.77^{\circ}(p=0.16)$. Similarly the SS for the reduction group changed from $43.08^{\circ}$ to $30.51^{\circ}$ ( $p=0.096$ ). Mean preoperative LL was $49.69^{\circ}$ overall, with a mean of in-situ group being $51.46^{\circ}$ and mean of the reduction group being $47.92^{\circ}(p>0.05)$. Mean LL for in-situ group changed from $51.46^{\circ}$ to $62.65^{\circ}$ $(p=0.08)$ and for reduction group from $47.92^{\circ}$ to $45.88^{\circ}$ $(p=1.00)$.

\section{6) Sagittal vertical axis}

Mean preoperative SVAs for groups A and B were +3.4 and $+3.2 \mathrm{~cm}$, respectively. Preoperative SVA evaluations were comparable with no significant difference between the groups $(p>0.05)$. Mean postoperative SVA was reduced in both groups; however, postoperative SVA changes were not significant $(p>0.05)$.

\section{Comparison of postoperative L5 measured spinopel- vic parameters between groups $A$ and $B$}

Postoperative spinopelvic parameters measured from the L5 between groups A and B were compared. Both groups showed a comparable reduction in PT and SFD with no significant difference ( $p>0.05$ for both). Dubousset's LSA increased in both groups, implying a reduction in LSK in both groups with no significant difference $(p>0.05)$ (Table 3).

When measuring PI from L5 after fusion, group A showed a significant decrease in PI to $41.33^{\circ}$. However, a similar change in PI was not seen in group B (mean postoperative $\left.\mathrm{PI}, 61.81^{\circ}\right)$. Comparing postoperative PI, a significant difference $(p=0.008)$ was noted between the groups. This difference was accompanied by a significant difference in SS while PT remained comparable for both groups. Postoperative SS was significantly higher in group B $\left(50.77^{\circ}\right)$ compared with group A $\left(30.51^{\circ}, p=0.0009\right)$. The large SS in the in-situ group was also accompanied by a significantly larger LL of $62.65^{\circ}$ compared to $45.88^{\circ}$ for the reduction group.

\section{Discussion}

Surgical management of HGS is controversial [1]. While anatomical reduction and fusion has been considered to be biomechanically advantageous, in-situ fusion has provided equivalent clinical outcomes in several studies [2-9]. It remains unclear how diametrically opposite treatment methodologies are effective. Spinopelvic parameters have gained credence recently in evaluating and planning management strategies for patients with HGS [10-12]. In our study, we observed that in-situ fusion and reduction techniques provide similar clinical outcomes. We also noted that spinopelvic parameters showed significant postoperative improvements when the L5 was considered the new sacrum, as it becomes part of the pelvis after fusion (Fig. 1). In our study, both groups were comparable for age, sex, grade of slip, and preoperative spinopelvic parameters. In both groups, considering the L5 as a part of the sacrum, a favorable change was noted in spinopelvic parameters of LSK, PT, and SFD following fusion. The decrease in PT, SFD, and LKS were observed to be closer to normal values described in the literature [12]. These findings provided an explanation based on spinopelvic parameters that in-si$t u$ fusion patients have comparable clinical and functional outcomes to those with severe spondylolisthesis undergoing reduction and fusion. In the case of a naturally occurring sacralized L5, pelvic parameters are measured from the L5. Similarly following L5-S1 fusion, the L5 becomes a part of the sacrum, and the L5 was used for measuring spinopelvic parameters after fusion.

Duval-Beaupere et al. [18] first introduced the concept of PI, following which others described the various pelvic parameters and their importance vis-à-vis sagittal balance [19]. Sagittal alignment parameters have been correlated with postoperative outcomes in adults with spinal deformities and restoration of sagittal balance by reducing the deformity has been correlated with improved outcomes $[20,21]$. However, this remains controversial as there has been no outright evidence to prove superior overall clinical outcomes [15].

Historically, in-situ fusion has been the gold standard 
for HGS management by virtue of the simplicity of the surgical technique and its relatively lower perioperative morbidity [22-24]. Therefore, it does appear that we must revisit pelvic parameter assessment in patients with HGS treated via in-situ fusion versus reduction and fusion.

The Spine Deformity Study Group has formulated a classification for HGS to plan treatment strategies $[11,17]$. HGS is classified into two subgroups: balanced and unbalanced/retroverted pelvis. As per their analysis, they recommended reduction and fusion only for patients with unbalanced pelvis. Here, we noted that three pelvic parameters significantly changed in both groups: PT, LSK, and SFD. The most significant change was noted in PT, with a $>50 \%$ decrease in the angle for both groups. PT is reduced as the superior endplate of the $\mathrm{L} 5$, which is closer to the femoral axis and subtends a smaller angle at the femoral head. Thus, PT is reduced and pelvic retroversion is corrected (Fig. 2). The reduction in pelvic retroversion or PT is the key to improvement of the sagittal spinopelvic balance $[11,17,20]$. This correction occurs even with insitu fusion when considering the L5 as the top of the new pelvis following fusion; consequently, in-situ fusion also provides satisfactory clinical results.

LSK is an important study parameter because it actually measures the L5 slope. While the anterior translation or slip of the L5 contributes little to the sagittal balance, the L5 slope could adversely affect it. Hence, normalization of the L5 tilt, and not the L5 slip, restores sagittal balance [17]. Considering LSK, which is now calculated between the posterior aspect of L5 and the L4 superior endplate, it shows an increase in the angle trending toward normalization of the spinopelvic parameters.. LSK was increased and the L5 slope was restored even in the presence of persisting slip in group B. This improvement in the L5 slope possibly improves the overall sagittal profile and results in good functional outcomes.

SFD, when measured from the posterior edge of the L5 to the femoral axis, resulted in a reduced distance compared with when it was measured from the S1. All these changes contributed to the new pelvis being more anteverted and balanced. These points offered a plausible explanation for the clinical improvement noted in each group and can be attributed to the normalization of these three pelvic parameters of LSK, SFD, and PT. The improvement in these measurements, which are comparable in both groups, may explain the equally good results seen in both groups.
Postoperative PT and SFD measured from the L5 showed a comparable reduction in both groups irrespective of surgical strategy. The reduction in PT implied a decrease in the pelvic retroversion, which is the ultimate objective of a reduction and fusion. That a similar change was noted in both groups when the parameters were assessed from the L5 offers a possible explanation for comparable clinical results seen in the series. The reduction in SFD reduces the moment arm and shear force acting across the slip and this decrease was noted to occur in both groups.

A high PI is associated with HGS and this is reflected in the preoperative PI values in this series. The postoperative PI decreased in group A, whereas no significant change was noted in group B. PI approximately amounted to the arithmetic sum of PT and SS. This higher PI in group B was associated with a significantly higher SS due to comparable postoperative PT in both groups. The postoperative SS was higher in group $\mathrm{B}$, which resulted in a higher LL for group B.

HGS may have a sagittaly unbalanced spine, which is reflected with positive mean SVA in this series. However, as the deformity is limited to a single spinal segment, most compensatory regional curves in the lumbar and cervical spine prevent a gross positive imbalance. Additionally, compensatory mechanisms in the hip limit excessive deviation of SVA, which probably explains the results of SVA evaluation in this series.

Limitations of this study include the small study population and the retrospective nature of data collection. The incidence of HGS would preclude the possibility of large sample size. A prospective analysis of a larger number of cases to test the hypothesis and assess the spinopelvic parameters, assuming L5 as a part of the sacrum postoperatively, would offer a more comprehensive statistical analysis and help explain the good results with in-situ fusion for high-grade slips.

\section{Conclusions}

Our hypothesis demonstrated that spinopelvic parameters favorably change when the L5 is considered the new sacrum following fusion in HGS, possibly explaining why reduction and in-situ fusion achieve satisfactory results. This questions the need for risky reduction procedures to establish normal pelvic parameters. Improvement in PT, SFD, and LSK when considering the L5 as the new sacrum 
correlates with good clinical and functional outcomes for both strategies.

\section{Conflict of Interest}

No potential conflict of interest relevant to this article was reported.

\section{Acknowledgments}

The project was funded by Ganga Orthopaedic Research \& Education Foundation, Coimbatore.

\section{References}

1. Cheung EV, Herman MJ, Cavalier R, Pizzutillo PD. Spondylolysis and spondylolisthesis in children and adolescents: II. surgical management. J Am Acad Orthop Surg 2006;14:488-98.

2. DeWald CJ, Vartabedian JE, Rodts MF, Hammerberg KW. Evaluation and management of high-grade spondylolisthesis in adults. Spine (Phila Pa 1976) 2005;30(6 Suppl):S49-59.

3. Harris IE, Weinstein SL. Long-term follow-up of patients with grade-III and IV spondylolisthesis: treatment with and without posterior fusion. J Bone Joint Surg Am 1987;69:960-9.

4. Lonstein JE. Spondylolisthesis in children: cause, natural history, and management. Spine (Phila Pa 1976) 1999;24:2640-8.

5. Sailhan F, Gollogly S, Roussouly P. The radiographic results and neurologic complications of instrumented reduction and fusion of high-grade spondylolisthesis without decompression of the neural elements: a retrospective review of 44 patients. Spine (Phila $\mathrm{Pa}$ 1976) 2006;31:161-9.

6. Freeman BL 3rd, Donati NL. Spinal arthrodesis for severe spondylolisthesis in children and adolescents: a long-term follow-up study. J Bone Joint Surg Am 1989;71:594-8.

7. Molinari RW, Bridwell KH, Lenke LG, Ungacta FF, Riew KD. Complications in the surgical treatment of pediatric high-grade, isthmic dysplastic spondylolisthesis: a comparison of three surgical approaches. Spine (Phila Pa 1976) 1999;24:1701-11.

8. Muschik M, Zippel H, Perka C. Surgical management of severe spondylolisthesis in children and adoles- cents: anterior fusion in situ versus anterior spondylodesis with posterior transpedicular instrumentation and reduction. Spine (Phila Pa 1976) 1997;22:203642.

9. Seitsalo S, Osterman K, Hyvarinen H, Schlenzka D, Poussa M. Severe spondylolisthesis in children and adolescents: a long-term review of fusion in situ. J Bone Joint Surg Br 1990;72:259-65.

10. Labelle H, Roussouly P, Chopin D, Berthonnaud E, Hresko T, O'Brien M. Spino-pelvic alignment after surgical correction for developmental spondylolisthesis. Eur Spine J 2008;17:1170-6.

11. Labelle H, Mac-Thiong JM, Roussouly P. Spinopelvic sagittal balance of spondylolisthesis: a review and classification. Eur Spine J 2011;20 Suppl 5:641-6.

12. Labelle H, Roussouly P, Berthonnaud E, et al. Spondylolisthesis, pelvic incidence, and spinopelvic balance: a correlation study. Spine (Phila Pa 1976) 2004;29:2049-54.

13. Bartolozzi P, Sandri A, Cassini M, Ricci M. One-stage posterior decompression-stabilization and transsacral interbody fusion after partial reduction for severe L5-S1 spondylolisthesis. Spine (Phila Pa 1976) 2003;28:1135-41.

14. Bridwell KH. Surgical treatment of high-grade spondylolisthesis. Neurosurg Clin N Am 2006;17:331-8.

15. Transfeldt EE, Mehbod AA. Evidence-based medicine analysis of isthmic spondylolisthesis treatment including reduction versus fusion in situ for high-grade slips. Spine (Phila Pa 1976) 2007;32(19 Suppl):S126-9.

16. Glavas P, Mac-Thiong JM, Parent S, de Guise JA, Labelle $\mathrm{H}$. Assessment of lumbosacral kyphosis in spondylolisthesis: a computer-assisted reliability study of six measurement techniques. Eur Spine J 2009;18:212-7.

17. Hresko MT, Labelle H, Roussouly P, Berthonnaud E. Classification of high-grade spondylolistheses based on pelvic version and spine balance: possible rationale for reduction. Spine (Phila Pa 1976) 2007;32:2208-13.

18. Duval-Beaupere G, Schmidt C, Cosson P. A Barycentremetric study of the sagittal shape of spine and pelvis: the conditions required for an economic standing position. Ann Biomed Eng 1992;20:451-62.

19. Legaye J, Duval-Beaupere G, Hecquet J, Marty C. Pelvic incidence: a fundamental pelvic parameter 
for three-dimensional regulation of spinal sagittal curves. Eur Spine J 1998;7:99-103.

20. Lenke LG, Bridwell KH. Evaluation and surgical treatment of high-grade isthmic dysplastic spondylolisthesis. Instr Course Lect 2003;52:525-32.

21. Ruf M, Koch H, Melcher RP, Harms J. Anatomic reduction and monosegmental fusion in high-grade developmental spondylolisthesis. Spine (Phila Pa 1976) 2006;31:269-74.

22. Burkus JK, Lonstein JE, Winter RB, Denis F. Longterm evaluation of adolescents treated operatively for spondylolisthesis: a comparison of in situ arthrodesis only with in situ arthrodesis and reduction followed by immobilization in a cast. J Bone Joint Surg Am 1992;74:693-704.

23. Poussa M, Remes V, Lamberg T, et al. Treatment of severe spondylolisthesis in adolescence with reduction or fusion in situ: long-term clinical, radiologic, and functional outcome. Spine (Phila Pa 1976) 2006;31:583-90.

24. Poussa M, Schlenzka D, Seitsalo S, Ylikoski M, Hurri $\mathrm{H}$, Osterman K. Surgical treatment of severe isthmic spondylolisthesis in adolescents: reduction or fusion in situ. Spine (Phila Pa 1976) 1993;18:894-901. 